\title{
INFLUÊNCIA DO SOMBREAMENTO NO CRESCIMENTO DE MUDAS DE CUMARU (Dipteryx odorata (AUBL.) WILLD. - FABACEAE), CULTIVADAS EM VIVEIRO
}

\author{
Toshihiro UCHIDA', Moacir A. A. CAMPOS ${ }^{2}$
}

RESUMO - Este trabalho apresenta os resultados de um experimento realizado com mudas de Dipteryx odorata (Aubl.) Willd. no viveiro da Estação Experimental de Silvicultura Tropical, do Inpa, em Manaus, Amazonas. O objetivo foi avaliar o desempenho da espécie cultivada em viveiro sob diferentes niveis de sombreamento. O experimento foi instalado em blocos ao acaso, com cinco tratamentos: dois tratamentos com sombra temporária, passando a pleno sol e três tratamentos com sombreamento de $30 \%, 50 \%$ e $70 \%$, obtidos com o uso de telas de poliolefinas, de cor preta. Aos 3 e 5 meses foram avaliados após a semeadura, diâmetro do colo, altura, comprimento das raizes, peso de matéria seca de raizes, caule e folhas e as relações altura/ diâmetro do colo e parte aérea/sistema radicular. Tanto aos 3 quanto aos 5 meses, o sombreamento não afetou o crescimento em altura e o comprimento das raízes. $\mathrm{O}$ diâmetro do colo foi maior sob $0 \%$ de sombreamento, sendo superior estatisticamente ao tratamento com $70 \%$ em ambos períodos. Com relação ao peso de matéria seca, o tratamento com $70 \%$ de sombreamento foi inferior aos demais na primeira medição. Na segunda medição verificou-se uma tendência de aumento do peso de matéria seca com a diminuição dos níveis de sombreamento. Os menores valores das relações altura/diâmetro do colo e parte aérea /sistema radicular foram obtidos a pleno sol. Dipteryx odorata, uma espécie típica das matas altas de terra-firme, cresce melhor a plena luz, quando cultivada em viveiro.

Palavras-chave: Amazônia, espécies florestais, viveiro

\section{Influence of Shade on the Growth of Cumaru (Dipteryx odorata (Aubl.) Willd. - Fabaceae) Seedlings in the Nursery}

ABSTRACT - This paper presents results of an experiment carried out in the nursery at the Tropical Silviculture Experiment Station of Inpa, in Manaus, Amazonas, Brazil, with Dipteryx odorata (Aubl.) Willd. seedlings. The objective was to assess the growth of the species in the nursery under different levels of shade. The experiment used fully randomised blocks with five treatments; two treatments with temporary shading which was removed later to provide full sun light $(0 \%)$ and three treatments under shade $(30 \%, 50 \%$ and $70 \%)$ using black polypropylene mesh. Stem diameter, height, root length, leaf, root, stem dry weights, shoot/root ratio and height/diameter ratio were assessed at three and five months after sowing. Height and root length were unaffected by shade levels at three and five months. Stem diameter was larger in full sun light, being statistically superior to the treatment with $70 \%$ shade, in both periods. Dry weight obtained under $70 \%$ shade was less than for other treatments at three months. At five months, there was a trend to increase dry weight as shade levels decreased. Smaller values of root/shoot ratio and height/diameter ratio were obtained in full sun. Dipteryx odorata, a species typical of the terra-firme (non-flooded) high forest, grows better under full sun light.

Key words: Amazônia, forest species, nursery

\section{INTRODUÇÃO}

O cumaru (Dipteryx odorata (Aubl.) Willd.) é uma Fabaceae, freqüente em toda Amazônia, nas matas de terra-firme, ocorrendo também no Mato Grosso, em Corumbá. Trata-se de uma árvore

\footnotetext{
'Agência de Cooperação Internacional do Japão - JICA

${ }^{2}$ Instituto Nacional de Pesquisas da Amazônia - INPA, Cx. Postal 478, 69011-970 Manaus, AM - Brasil
} 
grande, com $30 \mathrm{~m}$ de altura, cujas sementes aromáticas produzem um óleo essencial e industrial que a torna importante espécie do extrativismo (Lescure \& Castro, 1992). A madeira muito pesada $\left(0,95-1,00 \mathrm{~g} / \mathrm{cm}^{3}\right)$ e imputrescivel é tida como uma das melhores madeiras para dormentes, não apenas pela durabilidade, como também não se fende quando exposta ao sol. É utilizada também em implementos agrícolas, construção naval, cabos de ferramentas, moirões, carroçaria, estacas, esteios, eixo de moinhos, tacos para soalho (Loureiro et al., 1979).

O estudo da influência de fatores tais como densidade, substrato, água e luz é fundamental para o desenvolvimento de tecnologias de produção de mudas com alto padrão de qualidade. A luz é um dos principais fatores que influenciam o crescimento dos vegetais, por ser fonte primária de energia para a fotossintese. Neste aspecto, estudos sobre o efeito do sombreamento no crescimento e desenvolvimento de mudas cultivadas em viveiro foram realizados para Schefflera morototoni (Barbosa, 1985), Jacaranda copaia (Barbosa, 1990), Dinizia excelsa (Varela \& Santos, 1992), Licaria canela (Pinto et al., 1993), Ceiba pentandra (Pedroso \& Varela, 1995), Copaifera multijuga (Varela et al., 1995), Calophyllum angulare (Muroya et al., 1997). A despeito do grande número de espécies existente na Amazônia, a pesquisa florestal vem trabalhando com uma quantidade reduzida de espécies com valor comercial e de interesse silvicultural. Isto ocorre devido a aspectos estruturais da pesquisa como também a escassez e irregularidade no fornecimento das sementes.

Mudas desenvolvidas em viveiro podem receber condições ótimas para seu desenvolvimento inicial, aumentando as suas chances de se estabelecerem no campo. Mudas com alto padrão de qualidade tem maior possibilidade de originarem plantios sadios e mais produtivos. Todavia, para as espécies amazônicas, existe pouca informação sobre as técnicas de viveiro que produzem mudas mais vigorosas. Com o aumento da demanda por essas informações, principalmente em decorrência do avanço do desmatamento e multiplicação de áreas degradadas, é de vital importância que estudos sejam realizados visando contribuir com informações sobre as espécies nativas.

Com a implantação do Projeto Jacaranda (INPA/JICA), as pesquisas com mudas tiveram continuidade, visando atender a demanda de mudas a serem usadas na recuperação de áreas degradadas. $\mathrm{O}$ presente trabalho tem como objetivo avaliar o desempenho do cumaru (Dipteryx odorata) cultivado em viveiro sob diferentes níveis de sombreamento.

\section{MATERIAL E MÉTODOS}

O experimento foi implantado no viveiro de produção de mudas, da Estação Experimental de Silvicultura Tropical - EEST, do Instituto Nacional 
de Pesquisas a Amazônia - INPA. A EEST está localizada no $\mathrm{km} 45$ da BR174, Manaus - Boa Vista, ao Norte de Manaus. O clima da região de Manaus é do tipo Afi, segundo a classificação de Köeppen, apresentando pluviosidade de $2.485 \mathrm{~mm}$ e temperatura média anual de $25,6^{\circ} \mathrm{C}$, com estação seca de junho a outubro (Ribeiro, 1976).

As sementes de Dipteryx odorata foram coletadas de árvores portasementes no bairro Zumbi, zona Leste de Manaus, em 22/07/97. As sementes foram beneficiadas e postas para germinar em 24/07/97, diretamente em sacos de plástico com $22 \mathrm{~cm}$ de altura e $12 \mathrm{~cm}$ de diâmetro, contendo como substrato a mistura de barro, areia, esterco de galinha e palha de arroz carbonizada na proporção $12: 4: 0,5$ $: 1$, respectivamente.

Foram utilizados dois tratamentos com sombra temporária passando a pleno sol, e três tratamentos com sombra durante todo o periodo de observação, utilizando-se telas de poliolefinas, de cor preta (sombrite):

$\mathrm{T} 1$ = Pleno sol, após 48 dias sob duas camadas de sombrite com $50 \%$ de sombreamento

T2 = Pleno sol, após 65 dias sob $50 \%$ de sombreamento

$\mathrm{T} 3=30 \%$ de sombreamento

$\mathrm{T} 4=50 \%$ de sombreamento

$\mathrm{T} 5=70 \%$ de sombreamento

$\mathrm{O}$ delineamento experimental utilizado foi blocos ao acaso. As medições foram realizadas em dois períodos, nos dias 09/10/97 e 29/12/
97, cerca de 3 e 5 meses após a semeadura, respectivamente, utilizando-se um total de 20 mudas em cada periodo. Foram medidos o comprimento da parte aérea e do sistema radicular e o diâmetro do colo. As mudas foram colocadas para secar em estufa a $75^{\circ} \mathrm{C}$, por 72 horas e em seguida foram pesadas. A partir das variáveis obtidas foram calculadas as relações altura/diâmetro e parte aérea/ sistema radicular. Os dados foram submetidos a análise de variância e as médias comparadas pelo o teste de Tukey, ao nivel de $5 \%$ de probalidade.

\section{RESULTADOS E DISCUSSÃO}

As mudas de Dipteryx odorata apresentaram rápido crescimento em viveiro, atingindo $30 \mathrm{~cm}$ de altura em três meses. Entretanto, observou-se que não houve diferenças significativas entre as médias de altura e comprimento da raiz para os tratamentos utilizados, nem no terceiro, nem no quinto mês (Tab, 1). A altura das mudas na ocasião do plantio exerce importante papel na sobrevivência e desenvolvimento nos primeiros anos após o plantio (Carneiro, 1995). Barbosa (1990) não encontrou diferenças entre as médias de altura das mudas de Jacaranda copaia, cultivadas a pleno sol e com $30 \%, 50 \%$ e $70 \%$ de sombreamento. Da mesma forma, os níveis de sombreamento não influenciaram o crescimento em altura de mudas de Cedrelinga catenaeformis (Farias et al., 1997). Dipteryx odorata parece melhor adaptada à condições de pleno 
sol, ao contrário de Dinizia excelsa que cresceu melhor com $30 \%$ de sombra (Varela \& Santos, 1992) e Calophyllum angulare que apresentou maior desenvolvimento em altura sob $70 \%$ de sombra (Muroya et al., 1997).

Segundo Kozlowski (1962, citado por Engel, 1989), existe considerável evidência de que o crescimento em altura de mudas de muitas espécies ocorre primariamente às expensas de carbohidratos armazenados nas sementes. Por outro lado, as respostas das plantas às mudanças do ambiente também estão relacionadas a fatores genéticos de cada espécie. No caso das mudas de cumaru utilizadas neste experimento observou-se uma capacidade de adaptação aos diferentes níveis de sombreamento utilizados. Além disso, esta espécie apresenta semente volumosa e comprida com $2,6 \mathrm{~g}$ (Hidalgo, 1993), implicando em grande quantidade de reservas para o crescimento inicial das mudas.

Com relação ao diâmetro do colo, observou-se que o resultado obtido sob $70 \%$ de sombreamento foi estatisticamente inferior aos demais tratamentos (Tab. 1). Nota-se que o tratamento a pleno sol apresentou valores superiores aos tratamentos com $30 \%, \quad 50 \%$ e $70 \%$ de sombreamento, embora não existam diferenças significativas entre eles. Resultados similares foram encontrados por Barbosa (1990) e Varela \& Santos (1992), que observaram um decréscimo nos

Tabela 1. Médias de altura da parte aérea, comprimento da raiz, diâmetro do colo e razão altura/ diâmetro do colo (H/D) de mudas de Dipteryx odorata, cultivadas sob diferentes niveis de sombreamento.

\begin{tabular}{cccccccc}
\hline \multicolumn{3}{c}{$09 / 10 / 97$} & \multicolumn{5}{c}{$29 / 12 / 97$} \\
\hline Sombreamento & $\begin{array}{c}\text { Altura } \\
(\mathrm{cm})\end{array}$ & $\begin{array}{c}\text { Raiz } \\
(\mathrm{cm})\end{array}$ & $\begin{array}{c}\phi c \text { colo } \\
(\mathrm{mm})\end{array}$ & H/D & $\begin{array}{c}\text { Altura } \\
(\mathrm{cm})\end{array}$ & $\begin{array}{c}\phi c o l o \\
(\mathrm{~mm})\end{array}$ & H/D \\
$0 \%\left({ }^{\prime}\right)$ & 30,8 & 23,1 & $4,82 \mathrm{a}$ & $6,41 \mathrm{~b}$ & 41,8 & $6,1 \mathrm{a}$ & $6,85 \mathrm{~b}$ \\
$0 \%\left(^{2}\right)$ & 35,0 & 23,8 & $4,55 \mathrm{ab}$ & $7,74 \mathrm{a}$ & 42,7 & $6,0 \mathrm{a}$ & $7,09 \mathrm{ab}$ \\
$30 \%$ & 31,6 & 23,2 & $4,61 \mathrm{ab}$ & $6,92 \mathrm{ab}$ & 42,6 & $5,8 \mathrm{a}$ & $7,37 \mathrm{ab}$ \\
$50 \%$ & 34,9 & 20,7 & $4,53 \mathrm{ab}$ & $7,79 \mathrm{a}$ & 39,2 & $5,7 \mathrm{ab}$ & $6,88 \mathrm{ab}$ \\
$70 \%$ & 31,6 & 21,1 & $4,27 \mathrm{~b}$ & $7,41 \mathrm{ab}$ & 40,3 & $5,1 \mathrm{~b}$ & $7,75 \mathrm{a}$ \\
$\mathrm{F}$ & $2,8^{*}$ & $2,8^{*}$ & $3,2^{\star}$ & $4,99^{\star \star}$ & 1,07 & $5,56^{* *}$ & $2,82^{*}$ \\
\hline
\end{tabular}

(1) após 48 dias sob duas camadas de sombrite com $50 \%$ de sombreamento

(2) após 65 dias sob $50 \%$ de sombreamento

Médias seguidas da mesma letra não diferem estatisticamente entre si ao nivel de $5 \%$ de probabilidade, pelo teste de Tukey.

**Teste F significativo ao nivel de $1 \%$; "Teste F significativo ao nivel de $5 \%$. 
valores de diâmetro do colo em conseqüência do aumento do sombreamento. Entretanto, Muroya et al. (1997), estudando o crescimento de mudas de $C$. angulare sob diferentes niveis de sombreamento, registraram tendência inversa, ou seja, o aumento do sombreamento proporcionou maiores valores de diâmetro do colo. As mudas tem que apresentar um diâmetro do colo mínimo de acordo com a altura, pois existe uma forte correlação entre o diâmetro e a percentagem de sobrevivência após o plantio no campo (Carneiro, 1995).

$\mathrm{O}$ número e o peso de matéria seca de folhas não foram afetados pelo nivel de sombreamento na primeira medição (Tab. 2). Verificou-se que houve diferença significativa entre os tratamentos nos pesos de matéria seca de caule, raiz e total. O sombreamento de $70 \%$ reduziu o acúmulo de matéria seca em relação aos demais tratamentos. O peso de matéria seca do caule, raizes e total obtidos com $0 \%, 30 \%, 50 \%$ de sombreamento não apresentaram diferenças significativas entre si, entretanto percebe-se um tendência de aumento de peso de matéria seca com a diminuição do sombreamento.

Observou-se

diferenças

significativas entre os tratamentos para todas as características estudadas na segunda medição (Tab. 3). O número de folhas foi estatisticamente superior sob $30 \%$ de sombreamento, não havendo diferença significativa em relação ao pleno sol (após sombra temporária de $50+50 \%$ ). Com relação ao peso de matéria seca das folhas,

Tabela 2. Médias do número de folhas, peso de matéria seca de folhas, caule, raiz, matéria seca total e razão parte aérea/sistema radicular de mudas de Dipteryx odorata da primeira medição $(09 / 10 / 97)$.

\begin{tabular}{|c|c|c|c|c|c|c|}
\hline \multirow[b]{2}{*}{ Sombreamento } & \multirow[b]{2}{*}{$N^{\circ}$ de folhas } & \multicolumn{4}{|c|}{ Matéria seca $(\mathrm{g})$} & \multirow[b]{2}{*}{$S / F$} \\
\hline & & Folhas & Caule & Raiz & Total & \\
\hline $0 \%\left({ }^{1}\right)$ & 5,3 & 1,74 & $0,93 a b$ & $0,64 \mathrm{a}$ & $3,31 a b$ & 4,57 \\
\hline $0 \%\left({ }^{2}\right)$ & 5,4 & 1,94 & $1,06 \mathrm{a}$ & $0,62 \mathrm{a}$ & $3,61 a$ & 4,97 \\
\hline $30 \%$ & 5,3 & 1,86 & $0,97 a b$ & $0,56 a b$ & $3,42 a b$ & 4,98 \\
\hline $50 \%$ & 5,2 & 1,76 & $1,02 a b$ & $0,61 \mathrm{a}$ & $3,38 a b$ & 4,82 \\
\hline $70 \%$ & 4,75 & 1,52 & $0,79 b$ & $0,44 \mathrm{~b}$ & $2,76 b$ & 5,36 \\
\hline $\mathrm{F}$ & 1,91 & 1,37 & $3,11^{*}$ & $3,56^{\star \star}$ & $2,35^{*}$ & 1,06 \\
\hline
\end{tabular}

(1) após 48 dias sob duas camadas de sombrite com $50 \%$ de sombreamento (2) após 65 dias sob $50 \%$ de sombreamento

Médias seguida da mesma letra não diferem estatisticamente entre si ao nivel de $5 \%$ de probabilidade, pelo teste de Tukey.

${ }^{\star *}$ Teste F significativo ao nivel de 1\%; *Teste F significativo ao nivel de $5 \%$;

$S / R=$ Relação parte aérea / sistema radicular 
observou-se que o sombreamento de $30 \%$ forneceu o melhor resultado, embora não seja estatisticamente diferente dos tratamentos sem sombreamento. Isto sugere que o sombreamento reduziu o acúmulo de matéria seca nas folhas. O peso de matéria seca do caule foi favorecido pelos tratamentos sem sombreamento. Da mesma forma que o peso de matéria seca das folhas, observa-se que os valores obtidos decrescem com o aumento de sombreamento. Barbosa (1990) encontrou resultados semelhantes estudando mudas de Jacaranda copaia, onde o peso de matéria seca das folhas foi inferior no maior sombreamento. Em estudos realizados por Façanha \& Varela (1987) nenhuma diferença foi achada no acúmulo de matéria seca nas mudas de Eperua bijuga em função do sombreamento. Entretanto, para mudas de Dinizia excelsa, Varela \& Santos (1992) verificaram que os sombreamentos de 30 e $50 \%$ produziram maiores pesos de matéria seca da parte aérea.

$\mathrm{O}$ peso de matéria seca da raizes com $70 \%$ de sombreamento foi diferente estatisticamente dos demais. Os tratamentos com menor sombreamento favoreceram o desenvolvimento de raízes, de modo que menores níveis de sombreamento resultaram em maior peso de matéria seca de raizes (Tab, 3). Resultados semelhantes foram obtidos com mudas de Jacaranda copaia (Barbosa, 1990) e Dinizia excelsa (Varela \& Santos, 1992). Para mudas de Licaria canella, Pinto et al. (1993) observaram que o

Tabela 3. Médias do número de folhas, peso de matéria seca de folhas, caule, raiz, matéria seca total e razão parte aérea/sistema radicular de mudas de Dipteryx odorata da segunda medição $(29 / 12 / 97)$.

\begin{tabular}{ccccccc}
\hline & & \multicolumn{5}{c}{ Matéria seca $(\mathrm{g})$} \\
\cline { 3 - 7 } Sombreamento & $\mathrm{N}^{\circ}$ de folhas & Folhas & Caule & Raiz & Total & S/R \\
$0 \%\left({ }^{1}\right)$ & $8,0 \mathrm{ab}$ & $3,43 \mathrm{ab}$ & $2,77 \mathrm{a}$ & $2,25 \mathrm{a}$ & $8,45 \mathrm{a}$ & $2,83 \mathrm{c}$ \\
$0 \%\left({ }^{2}\right)$ & $7,2 \mathrm{~b}$ & $3,07 \mathrm{ab}$ & $2,56 \mathrm{ab}$ & $1,99 \mathrm{a}$ & $7,62 \mathrm{a}$ & $2,96 \mathrm{bc}$ \\
$30 \%$ & $8,4 \mathrm{a}$ & $3,69 \mathrm{a}$ & $2,46 \mathrm{ab}$ & $1,74 \mathrm{a}$ & $7,89 \mathrm{a}$ & $3,68 \mathrm{~b}$ \\
$50 \%$ & $6,9 \mathrm{~b}$ & $2,80 \mathrm{~b}$ & $2,13 \mathrm{bc}$ & $1,83 \mathrm{a}$ & $6,76 \mathrm{ab}$ & $2,83 \mathrm{c}$ \\
$70 \%$ & $7,1 \mathrm{~b}$ & $2,64 \mathrm{~b}$ & $1,82 \mathrm{c}$ & $1,08 \mathrm{~b}$ & $5,54 \mathrm{~b}$ & $4,57 \mathrm{a}$ \\
$\mathrm{F}$ & $4,6^{\star *}$ & $4,24^{\star *}$ & $6,13^{\star *}$ & $10,56^{\star *}$ & $6,39^{* *}$ & $15,17^{\star *}$ \\
\hline
\end{tabular}

(1) após 48 dias sob duas camadas de sombrite com $50 \%$ de sombreamento

(2) após 65 dias sob $50 \%$ de sombreamento

Médias seguida da mesma letra não diferem estatisticamente entre si ao nivel de $5 \%$ de probabilidade, pelo teste de Tukey

${ }^{\star \star}$ Teste F significativo ao nivel de $1 \%$;

$\mathrm{S} / \mathrm{R}=$ Relação parte aérea / sistema radicular 
tratamento sob $50 \%$ de sombreamento proporcionou maiores valores de matéria seca de raízes quando comparados com $0 \%$. Por outro lado a produção de matéria seca em mudas de Ceiba pentandra não foi influenciada pelos diferentes níveis de sombreamento utilizado (Pedroso \& Varela, 1995).

\section{CONCLUSÕES}

Concluiu-se que as mudas de Dipteryx odorata cultivadas em viveiro apresentam respostas diferentes em função dos diferentes niveis de sombreamento utilizados. Dipteryx odorata cresce melhor a pleno sol, na fase de viveiro, apresentando maior crescimento e mudas vigorosas com um bom padrão de qualidade. Nestas condições foram obtidos os maiores valores de diâmetro do colo, peso de matéria seca de raízes e parte aérea e consequentemente os menores valores para relação parte aérea/sistema radicular ( $\mathrm{S} / \mathrm{R}$ ) e relação altura/ diâmetro do colo (H/D). Houve uma tendência de acúmulo de matéria seca de folhas, caule e raizes com a diminuição dos níveis de sombreamento. $\mathrm{O}$ excesso de sombra ( $70 \%$ de sombreamento) prejudicou o crescimento das mudas.

\section{Bibliografia citada}

Barbosa, A.P. 1990. O crescimento de mudas de Caroba (Jacaranda copaia Aubl. D.Don.Bignoniaceae) cultivadas sob diferentes niveis de sombreamento e espaçamento. Anais do $6^{p}$ Congresso Florestal Brasileiro.
Sociedade Brasileira de Silvicultura. Campos do Jordão. p. 526-534.

Barbosa, A.P. 1985. Ecofisiologia do crescimento inicial de mudas de morototó (Schefflera morototoni, Aubl. Frondin Araliaceae) cultivadas sob quatro niveis de radiação solar e três niveis de espaçamento. Dissertação de Mestrado, Instituto Nacional de Pesquisas da Amazônia/Fundação Universidade do Amazonas. Manaus, Amazonas. 95p.

Carneiro, J.G.A. 1995. Produção e controle de qualidade de mudas florestais. Universidade Federal do Paraná/Fundação de Pesquisas Florestais, Curitiba, Paraná. $451 \mathrm{p}$.

Façanha, J.G.V.; Varela, V.P. 1987. Influência do tamanho da semente e tipo de sombreamento na produção de mudas de Muirapiranga. Pesq. Agropec. Bras., 22(11/12):1185-1189

Farias, V.C.; Varela, V.P.; Costa, S. de S.; Batalha, L.F.P. 1997. Análise de crescimento de mudas de cedrorana (Cedrelinga catenaeformis (Ducke) Ducke) cultivadas em condições de viveiro. Rev. Bras. de Sementes, 19 (2):193-200.

Hidalgo, F.A. Germinação e armazenamento de sementes de Diptervx odorata (Aubl.) Willd. - Fabaceae. Dissertação de Mestrado, Instituto Nacional de Pesquisas da Amazônia/Fundação Universidade do Amazonas. Manaus, Amazonas. 107p.

IBGE. 1991. Geografia do Brasil - Região Norte. Instituto Brasileiro de Geografia e Estatística, Rio de Janeiro. 307 p.

INPA/CPPF. 1991. Catálago de madeiras da Amazônia: caracteristicas tecnológicas; Area da hidrelétrica de Balbina. Instituto Nacional de Pesquisas da Amazônia. Manaus, Amazonas. 163 p.

Lescure, J.P.; Castro, A. 1992. L'extrativisme en Amazonie centrale. Bois et Foret des Tropiques, 231(1): 35-51.

Loureiro, A.A; Silva, M.F.; Alencar, J.C. 1979. Essências madeireiras da Amazônia. v.l. Instituto Nacional de 
Pesquisas da Amazônia. Manaus. 245 p.

Muroya, K.; Varela, V.P.; Campos, M.A.A. 1997. Análise de crescimento de mudas de jacareúba (Calophyllum angulare A. C. Smith-Guttiferae) cultivadas em condições de viveiro. Acta Amazonica, 27(3): 197212.

Pedroso, S.G.; Varela, V.P. 1995. Efeito do sombreamento no crescimento de mudas de sumaúma (Ceiba pentandra (L.) Gaertn.). Rev. Bras. de Sementes, 17(1): 47-51.

Pinto, A.M.; Varela, V.P.; Batalha, L.F.P. 1993. Influência do sombreamento no desenvolvimento de mudas de Louro pirarucu (Licaria canella (Meissn.) Kosterm.). Acta Amazonica, 23(4): $397-$ 404.

Ribeiro, M.N.G. 1976. Aspectos climatológicos de Manaus. Acta Amazonica, 6(2): 229-233,

Varela, V.P.; Santos, J. dos. 1992. Influência do sombreamento na produção de mudas de angelim pedra (Dinizia excelsa Ducke). Acta Amazonica, 22(3): 407-411.

Varela, V.P.; Vieira, M das G.G.; Melo, Z.L. de M. 1995. Influência do sombreamento sobre o crescimento de mudas de copaiba (Copaifera multijuga Hayne) e concentração de clorofila nas folhas. Boletim do Museu Paranaense Emilio Goeldi, 1I(2): 139-152. 